\title{
Surveillance of adverse events following H1N1/09 influenza immunisation in Victoria, Australia
}

\author{
David $\operatorname{Tran}^{1^{\star}}$, Hazel Clothier ${ }^{2,3}$, Jim P. Buttery ${ }^{2,4,5}$, Nigel W. Crawford ${ }^{1,2,6}$ \\ ${ }^{1}$ Department of General Medicine, Royal Children's Hospital (RCH), Melbourne, Australia; \\ *Corresponding Author: tran_haidavid@yahoo.com \\ ${ }^{2}$ SAEFVIC, Murdoch Childrens Research Institute (MCRI), Melbourne, Australia \\ ${ }^{3}$ School of Population Health, The University of Melbourne, Australia \\ ${ }^{4}$ Paediatric Infectious Diseases Unit, Monash Children's Hospital, Southern Health, Melbourne, Australia \\ ${ }^{5}$ Department of Paediatrics, Monash University, Melbourne, Australia \\ ${ }^{6}$ Department of Paediatrics, The University of Melbourne, Melbourne, Australia
}

Received 28 September 2012; revised 30 October 2012; accepted 12 November 2012

\section{ABSTRACT}

BACKGROUND: The H1N1 pandemic in 2009 required a systematic coordinated response, which in Australia included a monovalent (H1N1/09) vaccine (Panvax ${ }^{\circledR}$ ). SAEFVIC (Surveillance of Adverse Events Following Vaccination In the Community) is the Victorian, Australia state-based vaccine safety unit. The aim of the study was to review SAEFVIC reports of adverse events following immunisations (AEFI) temporally associated with H1N1/09 vaccines [monovalent and Trivalent Influenza Vaccines (TIV)]. METHODS: 1) Analysis of AEFI related to $\mathrm{H} 1 \mathrm{N1} / 09$ vaccines reported to SAEFVIC from September 2009 to December 2010; 2) Review of febrile convulsions (ICD-10 code R56.0), in children under 5 years of age presenting to the Royal Children's Hospital (RCH) Melbourne, Emergency Department between 1 March-30 April 2010. The presentation details and immunisation history were clarified by a telephone interview. RESULTS: 1) There were 659 reports of 749 adverse events following H1N1 vaccines. Among the TIV group, Fluvax had the most AEFI reported, with 77 per 100,000 doses distributed. Serious AEFI temporally associated with H1N1/09 vaccines included: 3 deaths, 2 anaphylactic reactions, and 3 GuillainBarre Syndrome. There were 7 reports of drug administration error; 2) There were 179 presentations with fever and 67 reported febrile convulsions out of 11025 presentations (0.61\%), 11 following H1N1 vaccines. Fluvax ${ }^{\circledR}$ was associated with $55 \%(6 / 11)$ reports. The mean onset time of AEFI was $\mathbf{1 3 . 2}$ hours post vaccination, and there was complete resolution of symptoms in allcases with no significant morbidity. CON-
CLUSION: Consistent with other Australian states in 2010, there was a TIV brand specific [Fluvax ${ }^{\circledR}$ ] increase in febrile convulsions post vaccination. As a result this vaccine is no longer licensed for children $<5$ years of age. Comprehensive passive and active surveillance for AEFI needs to be incorporated into future pandemic planning.

Keywords: H1N1; Influenza Vaccine; Adverse Events; SAEFVIC

\section{INTRODUCTION}

\subsection{HIN1/09 Pandemic \& Vaccine}

The emergence of an influenza A (H1N1) virus in 2009 raised widespread concern of a deadly pandemic as seen in 1918 [1]. Early surveillance data showed that children, young adults, pregnant women, obese patients and those with underlying pulmonary or cardiac diseases were more susceptible to the virus [2]. Within 4 weeks there were over 600 reported cases in Australia with a significant strain on primary health care providers. As part of the pandemic response, a monovalent influenza H1N1/09 vaccine (Panvax ${ }^{\circledR}$ CSL Ltd.) was produced and available 6-months post identification of the H1N1/09 strain. The aim of the pandemic immunisation program was to minimise the spread of the influenza virus and reduce the severity of the illness. Some of the issues encountered in development of the H1N1 vaccine included assessment of the immunogenicity of the vaccine, safety of the vaccine in human trials, sufficient production and distribution of the vaccine world-wide. In order to mass produce and rapidly distribute the vaccines, it was packaged in multi-dose vials (MDV) that contained thiomersal. There were also a number of potential adverse events following immunisation (AEFI) of interest, including 
Guillain-Barre Syndrome (GBS) which had an 8-fold relative incidence following a previous H1N1 influenza vaccine in the United States (1976-1977) [3,4].

The monovalent inactivated vaccine (Panvax ${ }^{\circledR}$ CSL Ltd.) was assessed for immunogenicity and safety in children and adults [5], and was made available for all Australians greater than 10 years of age on September 30th 2009 [6]. It was TGA approved for administration as a two-dose schedule in children under 10 years of age on December 3rd 2009. The monovalent vaccine was followed by a Trivalent Influenza Vaccine that was released in March 2010, in time for the Australian influenza season [see Figure 1]. As per WHO recommenda tions this seasonal influenza vaccine contained three strains: Influenza A/California/7/2009 (H1N1)-like virus; Influenza A/Perth/16/2009 (H3N2)-like virus and Influenza B/Brisbane/60/2008-like virus. Three brands were available in Australia in 2010: Fluvax ${ }^{\circledR}$ (CSL), Influvac ${ }^{\circledR}$ (Abbott) and Vaxigrip ${ }^{\circledR}$ (Sanofi Pasteur).

TIV brands were recommended for use in place of the monovalent Panvax ${ }^{\circledR}$ from March 2010 [7]. The monovalent vaccine, however, was free for all Australians and remained available throughout 2010. TIV was only funded for special risk groups and those $>65$ years of age [8]. An exception was the state of Western Australia, which had an annual funded TIV program for children $>6$ months to 5 years of age.

SAEFVIC (Surveillance of Adverse Events Following Vaccination in the Community) is the Victorian state based vaccine safety unit [9]. It was established in 2007 to enhance the monitoring of AEFI and provide clinical support and advice for immunisation providers. It relies on passive reporting of individual AEFI from Victorian Primary Health Care Providers and has a role in both the passive and active AEFI monitoring of new vaccines.

\subsection{Febrile Convulsions in Children}

The TGA established a separate reporting process for any AEFI post H1N1 vaccines, as part of the pandemic planning process [10]. Despite the mass program, the monovalent vaccine appeared well tolerated, with no safety issues flagged in the initial 3 - 4 months. Transition to the TIV in March 2010 was impacted by adverse events following immunisation (AEFI) reported in children, in particular febrile convulsions. This was predominantly in Western Australia, which as detailed above, was the only Australian state with a funded childhood influenza vaccine program. In other states TIV was funded for children with special risk conditions (e.g. pediatric cancer, cystic fibrosis) [8]. As a result, WA administered a much larger number of vaccines to children compared to other states (with a vaccine coverage of 52\%) [11], and was the earliest state to notice an increased in the numbers of febrile convulsions [12]. This resulted in the temporary suspension of all TIV administration to children under 5 years of age [13].

In response, on the 22nd April 2010, the Australian Health Department suspended all TIV for children under 5 year, pending further investigations being undertaken. The monovalent H1N1/09 influenza vaccine $\left(\right.$ Panvax $^{\circledR}$ ) remained available for this age group as no apparent increase in febrile convulsions above the expected rates had been observed. After an extensive investigation, that resulted in a similar vaccine suspension in New Zealand and delayed the FDA approval of the TIV in America and Europe, it was determined that the increased incidence of febrile convulsions was isolated to those that received the CSL Biotherapies vaccines (Fluvax ${ }^{\circledR}$ and Fluvax $\mathrm{Jr}^{\circledR}$ ). When the TIV suspension was lifted on July 30th 2010, it was recommended that only the Vaxigrip ${ }^{\circledR}$ and Influvac ${ }^{\circledR}$ vaccines be used for children less than 5 years old [14].

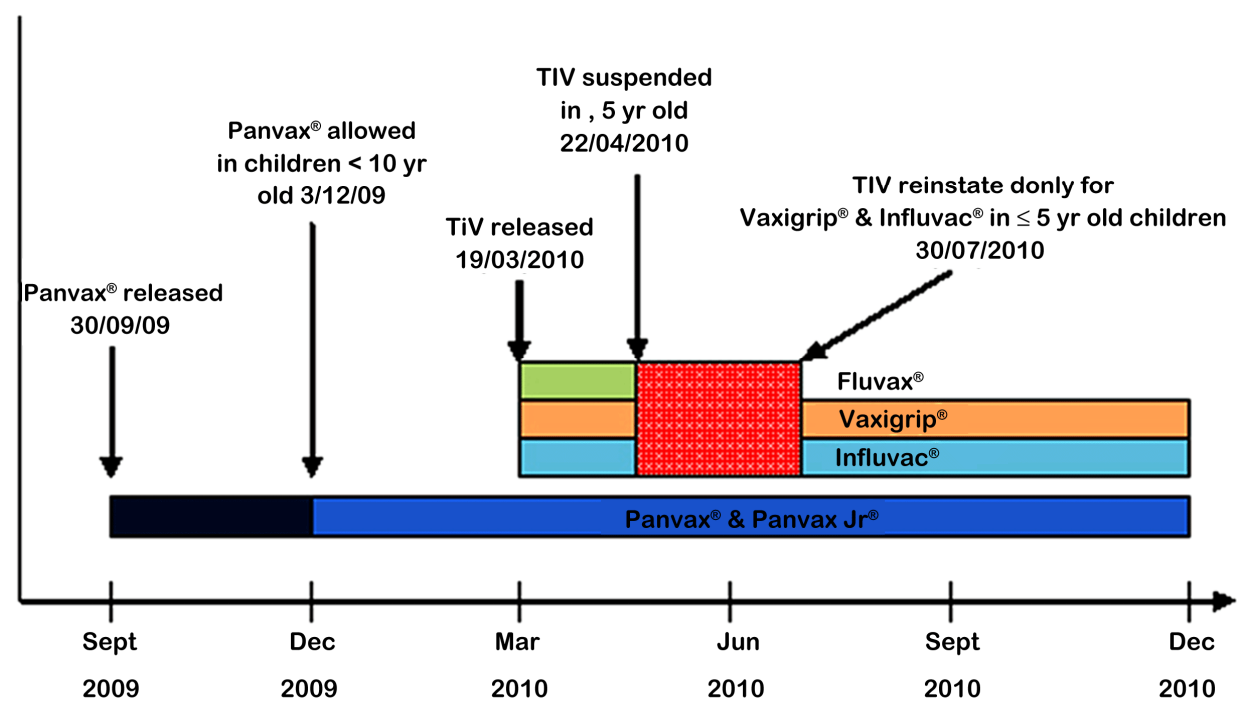

Figure 1. Timeline of release of H1N1 containing vaccines in 2009-2010. 


\subsection{AEFI Reporting in Australia}

Once concerns about the TIV vaccines' safety were raised, there were some delays in collating data from the individual states and territories. It highlighted some deficiencies in the co-ordination of monitoring for adverse reactions from vaccines. The Therapeutic Goods Administration was the national central reporting body, but information transfer between the states and this central body, was not as timely or complete as required. The Western Australian Stokes Report [15] published in July 2010 was an investigation into the surveillance of AEFI and is critical of the lack of clear reporting mechanisms. The report refers to the Victorian SAEFVIC reporting system [16] and identifies it as a benchmark for other states and territories in Australia.

\section{OBJECTIVE}

To analyse all adverse events following H1N1/09 monovalent and 2009-2010 seasonal trivalent influenza vaccine (TIV) as reported to the Victorian vaccine safety unit SAEFVIC.

\section{METHODS}

\subsection{Part A-Passive Surveillance for Adverse Events Following the Monovalent and Trivalent Influenza Vaccines (2009-2010)}

The SAEFVIC database was reviewed for all reports of AEFI received from September 30, 2009 to December 31, 2010. This time frame coincided with the introducetion of the H1N1 monovalent influenza vaccine (Pan$\operatorname{vax}^{\circledR}$ CSL), and incorporates the release of the seasonal TIV, Fluvax ${ }^{\circledR}$ (CSL), Influvac ${ }^{\circledR}$ (Abbott) and Vaxigrip ${ }^{\circledR}$ (Sanofi Pasteur), in March 2010 (Figure 1). These vaccines, as well as Panvax ${ }^{\circledR}$, were available until the end of 2010. Reports of AEFI were received by SAEFVIC through either by fax, post, or online [16]. Victorian reports from TGA and SAEFVIC were compared and crossreferenced.

All the AEFI reports were screened for any reports related H1N1/09. This included Panvax ${ }^{\circledR}$ and the seasonal TIV. Information was gathered regarding the co-administration of other vaccines, the onset of the reaction and the type of reaction. Estimates of the rates of adverse events were calculated as reports per 100,000 population using the estimated population of Victoria in June 2010 as 5.44 million [17].

Distributed dose data were provided by the Immunisation Section, Department of Health [Michael Batchelor Victorian Department of Health personal communication]. Administered dose data were not captured routinely on an immunisation register. Information on doses administered at a population level was obtained through representative surveys $[18,19]$. There was also some state based data from Western Australia [20]. Reports received were processed following SAEFVIC standard practices, which include review by an immunisation nurse and AEFI coding according to case definitions [9]. Each SAEFVIC report may have had several AEFI assigned.

Potential serious adverse events (defined as adverse events that resulted in death, ongoing sequalae, prolonged hospitalisation or requiring surgical intervention), were identified and detailed case reviews were performed. Of particular interest were neurological AEFI cases that included: febrile convulsions, Guillain-Barre Syndrome (GBS), transverse myelitis and temporal arteritis. Reports of drug administration errors were also noted. These adverse events were categorised into systems e.g. neurological, cardiovascular and respiratory. Adverse reactions were AEFI reactions were described according to age and vaccine brand administered. Analyses were conducted using Microsoft Excel [2010].

\subsection{Part B-Active Surveillance of Febrile Convulsions following Trivalent Influenza Vaccination}

Data were retrieved from the Emergency Department HAS database (Victorian Emergency Minimum Dataset) at the Royal Children's Hospital, Victoria. Data were extracted on May 4, 2010, and screened for presentations from March 1, 2010 to April 30, 2010, for any of the following ICD-10-AM codes: fever (R50.9), convulsions (R56.9) and febrile convulsions (R56.0). Patients were contacted via phone and were asked for information regarding the febrile convulsions, any preceding vaccinetions and the outcomes. These data were subsequently supplied to Governmental Health Department during the investigation into incidences of febrile convulsions related to vaccines [15]. RCH Human Research Ethics approval was obtained for the study [HREC 30052A].

\section{RESULTS}

\subsection{Part A}

From 30th September 2009 to 31st December 2010 SAEFVIC received 2401 reports of AEFI; 61\% females and 39\% males. Twenty-seven percent (659/2401) of reports were following vaccines that contained H1N1/09, an incidence of 12.1 per 100,000 population [17]. Of these, 9\% (59/659) were associated with co-administration of other vaccines.

Average time to onset of symptoms was 8 hours, with a range from 1 minute to 52 days following the immunisation. The majority of reactions (96.4\%) were following the initial vaccination dose. Distribution of vaccine brands varied widely throughout Australia. Distribution of vac- 
cine brands within Victoria from September 2009-December 2010 is illustrated in Table $\mathbf{1}$.

From the 659 reports, 749 reactions were identified (as a person may experience multiple reactions). Rates of AEFI by vaccine type are detailed in Table 1, with the highest rate observed post Fluvax ${ }^{\circledR}$, with 77.4 AEFI reactions per 100,000 doses distributed. The results by age group are detailed in Figure 2, with the highest in the 0 4 years group with 83.5 reports per 100,000 population. The proportion of AEFIs per age category are illustrated in Figure 3. The total number of AEFI by vaccine brand and system involved are detailed in Figure 4.

\subsubsection{Drug Errors in Administration of Vaccine}

There were 7 cases of human error in administering the vaccine (coded as "drug error"), of which 5 were from accidental injection of more than the $0.5 \mathrm{~mL}$ recommended dose (varied from twice to 10 times the recommended volume). Two reports were due to incorrect injection site and inadvertent administration of a 2nd

Table 1. Numbers of vaccine doses distributed from September 2009-December 2010 and the rates of AEFI reported per 100,000 doses of vaccine distributed.

\begin{tabular}{ccc}
\hline $\begin{array}{c}\text { Brand of H1N1 } \\
\text { containing } \\
\text { vaccine }\end{array}$ & $\begin{array}{c}\text { Number of doses } \\
\text { distributed }\end{array}$ & $\begin{array}{c}\text { Rate of AEFI per } \\
\mathbf{1 0 0 , 0 0 0} \text { doses of } \\
\text { vaccine }\end{array}$ \\
\hline Fluvax $^{\circledR}$ & 479,035 & 77.4 \\
Vaxigrip $^{\circledR}$ & 256,060 & 7.9 \\
Influvac $^{\circledR}$ & 202,363 & 16.0 \\
Panvax $^{\circledR}$ & $1,591,152$ & 20.2 \\
Total & $\mathbf{2 , 5 2 8 , 6 1 0}$ & \\
\hline
\end{tabular}

dose of vaccine on the same day. Only one drug error report resulted in an AEFI, which was a flu-like illness for 24 hours following the vaccine.

\subsubsection{Serious Reports of Adverse Events Following Immunisation}

1) Death

Three deaths were reported, all temporally associated with H1N1 containing vaccines ( 0.11 per 100,000 doses distributed). Two were deemed unrelated to the vaccine, with one case a Staphylococcal aureus sepsis and another mortality due to cardiac failure. A third case involved an 85 year-old man who died in a single motor vehicle accident 30 minutes after receiving the Fluvax ${ }^{\circledR}$. It was postulated that a vasovagal reaction post immunisation may have caused the accident and subsequent death, and resulted in reinforcement of the Australian Immunisation Handbook's recommendation that all patients wait for 30 minutes post before driving or using heavy equipment [8].

2) Anaphylaxis

There were 2 reported cases of anaphylaxis, both following the Panvax ${ }^{\circledR}$ vaccine. Both cases recovered completely following appropriate medical intervention including adrenaline therapy.

3) Guillain-Barre Syndrome

There were 3 AEFI reports of Guillain-Barre Syndrome. All 3 cases involved male patients over the age of 65 , following receipt of the Panvax ${ }^{\circledR}$ vaccine. All recovered completely following medical treatment.

4) Transverse Myelitis

There were 2 reports of Transverse Myelitis, both involving young adult patients following the Panvax ${ }^{\circledR}$ vac-

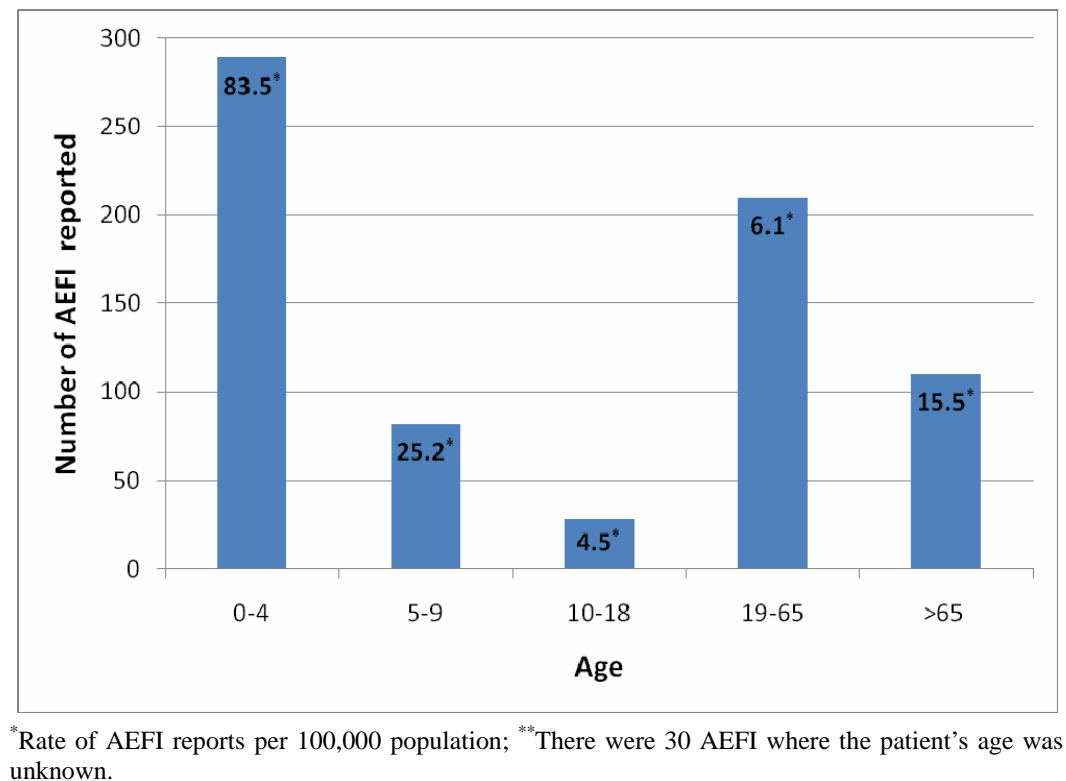

Figure 2. Rates of AEFI reports per 100,000 population by age ${ }^{* *}$ (years). 


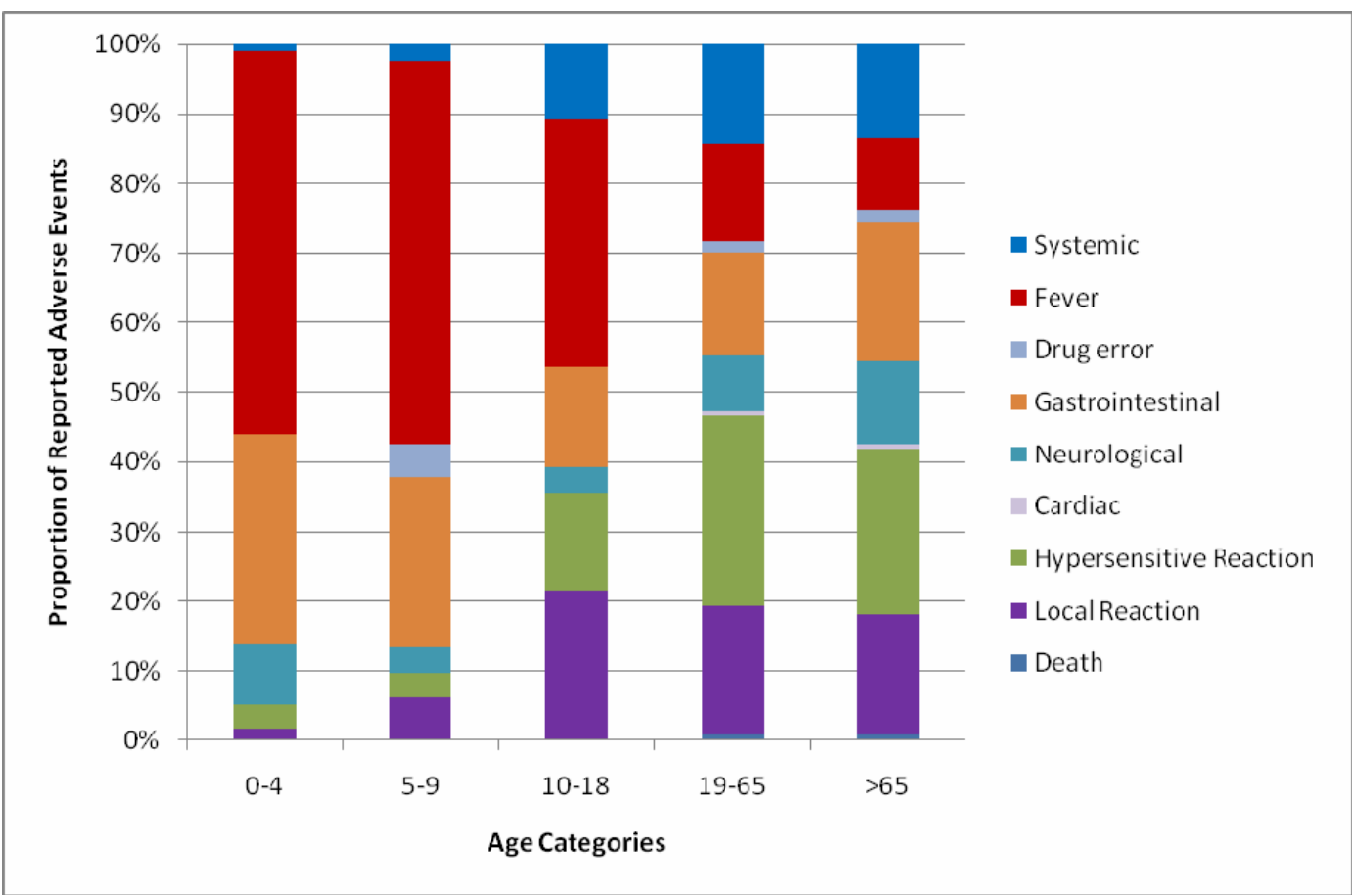

Figure 3. Adverse events reported by affected system and age group (years).

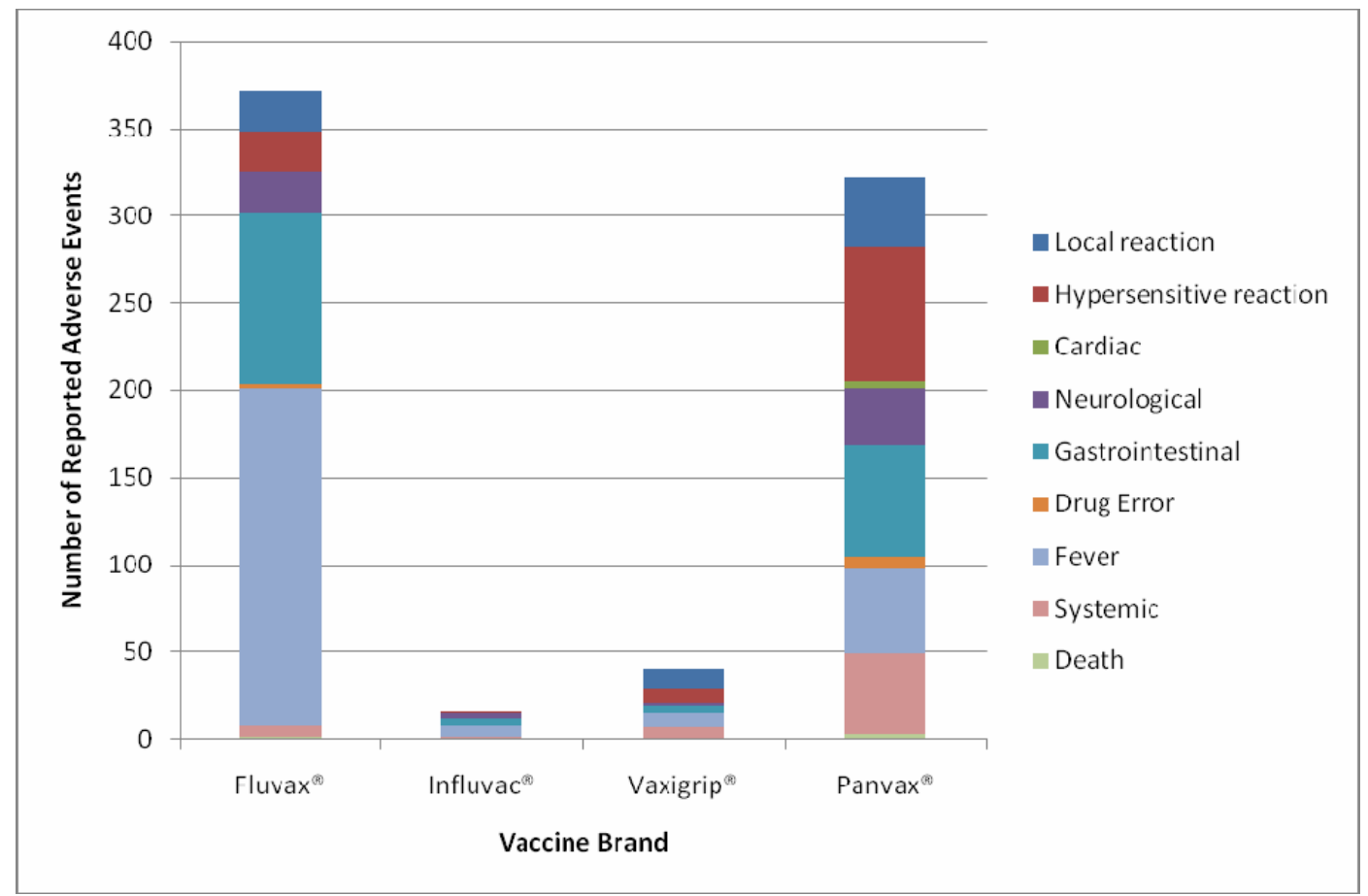

Figure 4. Adverse events reported by vaccine brand and affected systems.

cine. One patient had ongoing neurological deficits 2months post diagnosis, the second case completely recovered.

5) Cardiac Symptoms

There were 2 cases of cardiac arrhythmias and 1 of Angina. All occurred following the Panvax ${ }^{\circledR}$ vaccine.
The reports of cardiac arrhythmias were temporally associated with the Panvax ${ }^{\circledR}$ vaccine, but not suggestive of causation. Both were delayed reactions (one at 5 days, another at 2 weeks post vaccine). The case of cardiac angina was in a 75 year-old female who had pre-existing cardiac disease, who within 1 - 2 hours of receiving Pan- 
$\operatorname{vax}^{\circledR}$ vaccine developed angina, fever and headaches and aches and pains. This was treated with oral medication with complete recovery.

6) Fever

There was a total of 257 reports of fever, 21 were not measured and 236 were over $38^{\circ} \mathrm{C}$ (4.3/100,000 population). A majority of reports were in the paediatric population, with 159 reports in children under 5 years of age.

\subsection{Part B}

Active review of a single tertiary hospital, the RCH ED database from 1st March 2010-30th April 2010, saw 179 presentations of fever and 67 presentations with febrile convulsions. This was consistent with fever and febrile convulsion rates seen in a similar time frame in 2008-2009 (Table 2). There were 11 cases of febrile convulsions associated with H1N1 containing vaccines. Fluvax ${ }^{\circledR}$ accounted for 6 cases and Panvax ${ }^{\circledR}$ for 5 . In 3 of the reports, there were other vaccines administered at the same time as the H1N1/09 containing vaccine, including varicella vaccine (2) and pneumococcal conjugate vaccine (1).

Incorporating these data with the passive surveillance results received from the SAEFVIC database from September 30th 2009-December 31st, 2010, there were 21 reports of febrile convulsions following an H1N1 containing vaccine. A high proportion was following Flu$\operatorname{vax}^{\circledR}(14 / 21 ; 67 \%)$, with only 6 cases after Panvax ${ }^{\circledR}(6 / 21$; 29\%) and 1 following Influvac ${ }^{\circledR}$ (1/21; 4\%) (Table 3).

Of the patients who had a febrile convulsion, 20 presented to ED, and 17 were discharged within 24 hours. There was a 20 month old boy who initially presented to ED after a $<5$ minute generalised clonic tonic seizure (GTCS), however had 2 subsequent brief GTCS in the department. He was treated with intravenous midazolam and antibiotics for suspected sepsis. He was discharged 3 days after his febrile convulsions once the cultures were negative. Another patient had a known seizure disorder, and after a 20 minute febrile seizure was administered midazolam at home with subsequent seizure cessation. In a third case, a 20-month-old girl with no previous history of seizures had a 20-minute seizure at home, which ceased following rectal diazepam administered by the ambulance officers. She was observed in hospital and had normal baseline blood investigations. In the other remaining cases, there was no report of active treatment required for the febrile seizure, nor any long-term neurological sequelae reported.

\section{DISCUSSION}

The overall incidence of AEFI reported to SAEFVIC for H1N1/09 containing vaccines was 12.1 per 100,000 population. This was similar to the national reports to the TGA, quoted as 11 per 100,000 population [21]. The majority of adverse events were reported in the paediatric population, in particular in under 5 year-old patients at a rate of 83 per 100,000 population. This was much higher than any other age category, and is likely to be related to the febrile convulsions with TIV and subsequent reporting. Similar reporting rates were reported in national data where the paediatric population had highest numbers of reported AEFI-92.1 per 100,000 population in the less than 1 year range and 27.2 in the 1 to 2 age range [21]. When compared to international published data, the number of reported AEFI was higher in Australia. The US had 11.1 reports per 100,000 for the monovalent H1N1 vaccine (82 million doses administered) and 4.46 per 100,000 for the seasonal TIV (130.4 million doses) [22], and China had reporting rates of 9 per 100,000 (89.6 million doses administered), with only 13 per 100,000 in the $<10$ years old category [23].

Having a concomitant vaccine at the same time as the

Table 2. Presentations to RCH ED with diagnosis of fever (ICD-10-AM R50.9) and febrile convulsion (ICD-10-AM R56.0).

\begin{tabular}{cccccc}
\hline 1 Mar to 30 Apr & Fever & Rate of fever $^{*}$ & Febrile convulsions & Rate of febrile convulsions $^{*}$ & Total ED presentations $^{10394}$ \\
\hline 2008 & 139 & 13.4 & 61 & 5.9 & 4.5 \\
2009 & 107 & 10.0 & 48 & 6.1 & 10649 \\
2010 & 179 & 16.2 & 67 & 11025 \\
\hline
\end{tabular}

*Rate per 1000 presentations.

Table 3. Fever and febrile convulsions in children $<5$ years of age by vaccine brand.

\begin{tabular}{cccc}
\hline & Fluvax $^{\circledR}$ & Influvac $^{\circledR}$ & Panvax $^{\circledR}$ \\
\hline Fever & 138 & 2 & 19 \\
Febrile convulsions & 14 & 1 & 6 \\
Rate of Feb Convulsions $^{*}$ & 2.9 & 0.5 & 0 \\
\hline
\end{tabular}

*Rate per 100,000 doses distributed. 
H1N1 containing vaccine contributed to almost $10 \%$ of reported AEFI. There was a higher number of reported AEFI following the trivalent vaccines compared to the monovalent Panvax ${ }^{\circledR}$. This was particularly evident when corrected for the number of vaccine doses distributed (5.87 vs. 0.76 per 100,000 doses). The predominant reactions were of fever, local injection site reactions and vomiting. Within the $<5$ years old population, $55 \%$ of AEFI were due to fevers and $30.1 \%$ from gastrointestinal symptoms. This differed from the adult population where adverse events were more evenly distributed between local, hypersensitive, gastrointestinal and systemic reactions.

With the early release of the monovalent Panvax ${ }^{\circledR}$ vaccine in multi-dose vials that contained Thiomersal, there was criticism over the potential drug errors during administration [24]. This was reflective of the 5 out of 7 drug errors reported, that were associated with excessive doses administered of the Panvax ${ }^{\circledR}$ vaccine. Reports of serious AEFI were low and reflected similar numbers to previous years' vaccine releases [21]. SAEFVIC only had a small number of GBS cases reported, which historically has been of particular interest due to the increased incidence of GBS following the 1976 H1N1 National Influenza Vaccination Program in the US. From the SAEFVIC reports there were 3 cases of GBS ( 0.1 per 100,000 vaccinees), lower than the worldwide estimated incidence of GBS of 0.6 - 4.0 cases per 100,000 [25,26]. This compares with similarly low rates in US from 1990-2003 where GBS rates following influenza vaccine were reported to be 0.04 per 100,000 vaccinees [4].

Limitations to this study included the fact that SAEFVIC is a passive surveillance system [9]. Although all efforts were made to validate reported events; reporting bias, underreporting and inconsistency can impact on the data quality. The reported AEFI were temporally associated with the vaccine, but this does not apply causation. The timing post the vaccine is important as is the application of AEFI definitions such as the Brighton collabo ration criteria [9].

Extensive media coverage would also have skewed reporting numbers and types, and may have contributed to the high incidence of reported fevers and febrile convulsions in children in 2010.

Another major limitation was not having exact numbers of vaccine doses administered, which made calculations of reporting rates difficult. These were estimated using doses distributed rather than doses administered. This was particularly relevant in the case of the multidose vials where in many instances the whole vial was not used, and some doses were discarded or wasted [24]. Any future pandemic should ensure there is a vaccine register to better document doses given and provide the possibility of data-linking with adverse event databases such as SAEFVIC. The distributed doses could be determined up until end of 2010 (personal communication Michael Batchelor, Depatment Health Victoria, Immunisation Section). This data was used to compare the rates of AEFI between the different vaccines. A more accurate assessment of comparative rates would be obtained by using administered doses.

\section{Implications for Future Pandemics}

In 2011, the Department of Health and Ageing released a report into Australia's overall response to the H1N1 2009 pandemic [27]. It made recommendations in several areas such as communication, surveillance and vaccination planning. It emphasised the need to establish an easier, more centralised and more up-to-date reporting system with real-time data. The Stokes review of the Western Australian public health response to adverse events following the seasonal influenza vaccine recommended increased transparency between the State and Commonwealth Governments, establishing a web based reporting system such as that used by SAEFVIC to share information between the local and national departments. In addition, a national web based reporting system to record the number of vaccinations administered, and a form of early warning or "flag" system be established in order to alert any AEFI that occur in a pattern outside of norm [15]. This would be of particular use in detailing incidences of uncommon, serious vaccine related conditions. Conditions such as Guillain-Barre Syndrome are easily lost in passive reporting systems due to their low incidence rate, and only through active surveillance of hospital admissions is it possible to analyse any potential causal association [28]. The benefit of clinical follow-up at SAEFVIC has also been highlighted in reviews of anaphylaxis, with clinical details often insufficient to apply the Brighton criteria and confirm the diagnosis [29].

In regards to febrile convulsions post Fluvax ${ }^{\circledR}$, the TGA review (Horvath report) noted there was no safety signal from previous years' seasonal influenza vaccines or the Panvax ${ }^{\circledR}$ program [30]. It concludes that the actions to suspend the seasonal TIV for $<5$ years old children occurred in a timely and appropriate manner, but also makes recommendations for a model of vaccine safety monitoring, and more robust reporting systems to define protocols for management and program action in the event of a possible safety signal. Subsequent studies reviewing the incidence of fever and febrile convulsion associated with the seasonal influenza vaccine as well as reviewing potential reasons why Fluvax ${ }^{\circledR}$ was associated with the high incidence of fever and febrile convulsions without an underlying cause being identified Hypotheses have included: individuals' responses to the Fluvax ${ }^{\circledR}$ vaccine causing rapid pyrogenic cytokine responses; 
CSL's use of deoxycholateas a virus-splitting agent being linked with higher fevers; or problems with the vaccine storage itself [29,31].

The implications for the national immunisation program have been significant and this study contributed to some of the data incorporated into these national recommendations. In 2010 the outcome on the seasonal TIV recommendations was that Fluvax ${ }^{\circledR}$ was suspended indefinitely for children aged under 5 . The ban on the other TIV brands Influvac ${ }^{\circledR}$ and Vaxigrip ${ }^{\circledR}$ was lifted on 30 July 2010, and the Australian Technical Advisory Group on Immunisation (ATAGI) have recommended these vaccines in children aged 6 months - 5 years. ATAGI have also recommended these 2 brands for children aged 5 - 10 years of age, however Fluvax ${ }^{\circledR}$ may be used if no other alternative is available [32]. The US and Europe also suspended Fluvax ${ }^{\circledR}$ for use in children [33]. This continued into 2011-12 with Fluvax ${ }^{\circledR}$ not licensed for children under 5 years and not recommended in children less than 10 years of age.

\section{CONCLUSION}

Comprehensive documentation of AEFI post pandemic H1N1/09 vaccines was important and lessons learnt should be incorporated into future pandemic planning. Consistent with other Australian states, in 2010 SAEFVIC identified a brand specific [Fluvax ${ }^{\circledR}$ ] increase in febrile convulsions post vaccination. This SAEFVIC data was included in the national investigation and as a result Fluvax $^{\circledR}$ is currently not licensed for children less than 5 years of age. AEFI can have an impact on the public confidence in vaccines and this study highlights the importance of ongoing surveillance at both a state and national level

\section{ACKNOWLEDGEMENTS}

Thanks to all the SAEFVIC team.

\section{CONFLICT OF INTEREST}

NWC and JPB have acted as chief investigators for epidemiological studies sponsored by vaccine manufacturers (CSL) and serological testing (Merck). All payments, including for sitting on advisory boards (NWC), data safety monitoring boards (JPB), lecturing (NWC) and travel expenses for attendance at scientific meetings, are paid directly to an administrative fund held by Murdoch Childrens Research Institute.

\section{REFERENCES}

[1] Patterson, K.D. and Pyle, G.F. (1991) The geography and mortality of the 1918 influenza pandemic. Bulletin of the History of Medicine, 65, 4-21.

[2] Peiris, J.S., Poon, L.L. and Guan, Y. (2009) Emergence of a novel swine-origin influenza A virus (S-OIV) H1N1 virus in humans. Journal of Clinical Virology, 45, 169-173. doi:10.1016/j.jcv.2009.06.006

[3] Souayah, N., et al. (2007) Guillain-Barre syndrome after vaccination in United States a report from the CDC/FDA Vaccine Adverse Event Reporting System. Vaccine, 25, 5253-5255. doi:10.1016/j.vaccine.2007.03.053

[4] Haber, P., et al. (2004) Guillain-Barre syndrome following influenza vaccination. Journal of the American Medical Association, 292, 2478-2481. doi:10.1001/jama.292.20.2478

[5] Nolan, T., et al. (2010) Immunogenicity of a monovalent 2009 influenza A(H1N1) vaccine in infants and children: A randomized trial. Journal of the American Medical Association, 303, 37-46. doi:10.1001/jama.2009.1911

[6] Therapeutic Goods Administration (2009) TGA approves vaccine against Pnademic (H1N1) 2009 influenza. http://www.tga.gov.au/safety/alerts-medicine-pandemic-i nfluenza-090918.htm

[7] Department of Health Ageing (2010) The Facts on swine flu. D.o.H. Ageing, Canberra.

http://www.health.gov.au/internet/ministers/publishing.ns f/Content/mr-yr10-nr-nr053.htm

[8] National Health and Medical Research Council (2008) The Australian Immunisation Handbook. 9th Edition, National Capital Press, Canberra.

[9] Clothier, H.J., et al. (2011) Surveillance of adverse events following immunisation: The model of SAEFVIC, Victoria. Communicable Diseases Intelligence, 35, 294-298.

[10] Therapeutic Goods Administration (2010) Overview of vaccine regulation and safety monitoring and investigation into adverse events following 2010 seasonal influenza vaccination in young children.

http://www.tga.gov.au/pdf/alerts-medicine-seasonal-flu-1 01008.pdf

[11] Van Buynder, P.G., et al. (2011) Marketing paediatric influenza vaccination: Results of a major metropolitan trial. Influenza and Other Respiratory Viruses, 5, 33-38. doi:10.1111/j.1750-2659.2010.00167.x

[12] Armstrong, P.K., et al. (2011) Epidemiological study of severe febrile reactions in young children in Western Australia caused by a 2010 trivalent inactivated influenza vaccine. BMJ Open, 1, e000016. doi:10.1136/bmjopen-2010-000016

[13] Bishop, J. (2010) Seasonal influenza vaccine and young children. D.o.H.a. Ageing, Canberra.

[14] Therapeutic Goods Administration (2010) Investigation into Febrile Convulsions in young children after Seasonal Influenza Vaccination. Latest findings and recommendations 30th July 2010. D.o.H.a. Ageing, Canberra.

[15] Stokes, B. (2010) Ministerial review into the public health response into the adverse events to the seasonal influenza vaccine. D.o.H. Government of WA, Canberra.

[16] Surveillance of adverse events following vaccination in the community. SAEFVIC adverse events reporting, 2007. http://www.saefvic.org.au

[17] Australian Bureau of Statistics (2008) Population projections Australia 2006-2101. 
http://www.abs.gov.au/ausstats/abs@.nsf/detailspage/322 2.02006\%20to\%202101?opendocument.

[18] Australian Institute of Health and Wellbeing (2011) 2009 adult vaccination survey: Summary results. Cat. no. PHE 135.

http://www.aihw.gov.au/publication-detail/?id=10737418 409

[19] Australian Institute of Health and Wellbeing (2010) 2010 pandemic vaccination survey: Summary results. Cat. no. PHE128.

http://www.aihw.gov.au/workarea/downloadasset.aspx?id $=6442460016$

[20] Mak, D.B., et al. (2010) Pandemic (H1N1) 2009 influenza vaccination coverage in Western Australia. Medical Journal of Australia, 193, 401-404.

[21] Mahajan, D., et al. (2010) Annual report: Surveillance of adverse events following immunisation in Australia, 2009. Communicable Diseases Intelligence, 34, 259-276.

[22] Vellozzi, C., et al., (2010) Adverse events following influenza A (H1N1) 2009 monovalent vaccines reported to the Vaccine Adverse Event Reporting System, United States, October 1, 2009-January 31, 2010. Vaccine, 28, 7248-7255. doi:10.1016/j.vaccine.2010.09.021

[23] Liang, X.F., et al. (2011) Safety of influenza A (H1N1) vaccine in postmarketing surveillance in China. The New England Journal of Medicine, 364, 638-647. doi:10.1056/NEJMoa1008553

[24] Gosbell, I.B., et al. (2010) Immunisation and multi-dose vials. Vaccine, 28, 6556-6561. doi:10.1016/j.vaccine.2010.07.052

[25] Evans, D., Cauchemez, S. and Hayden, F.G. (2009) "Prepandemic" immunization for novel influenza viruses, "swine flu" vaccine, Guillain-Barre syndrome, and the detection of rare severe adverse events. The Journal of

\section{ABBREVIATIONS}

AEFI: Adverse events following immunisation; ATAGI: Australian technical advisory group on immunisation; GBS: Guillain-barre syndrome; ED: Emergency department; HPV: Human papillomavirus;
Infectious Diseases, 200, 321-328. doi:10.1086/603560

[26] Hughes, R.A. and Cornblath, D.R. (2005) Guillain-Barre syndrome. Lancet, 366, 1653-1666. doi:10.1016/S0140-6736(05)67665-9

[27] Department of Health Ageing (2011) Review of Australia's health sector response to pandemic (H1N1) 2009, lessons identified. D.o.H.a, Canberra.

[28] Crawford, N.W. (2011) GBS active: Targeted active surveillance for Guillain-Barre syndrome following the introduction of H1N1 vaccines in victoria, Australia. World Society of Paediatric Infectious Diseases Conference, Melbourne, 16-19 November 2011.

[29] Blyth, C.C., et al. (2011) Trivalent influenza vaccine and febrile adverse events in Australia, 2010: Clinical features and potential mechanisms. Vaccine, 29, 5107-5113. doi:10.1016/j.vaccine.2011.05.054

[30] Horvath, J. (2010) Review of the management of adverse events associated with Panvax and Fluvax. D.o.H. Ageing, Canberra.

[31] Kelly, H.A., et al. (2011) Adverse events associated with 2010 CSL and other inactivated influenza vaccines. Medical Journal of Australia, 195, 318-320. doi:10.5694/mja11.10941

[32] Department of Health Ageing (2010) Summary of ATAGI recommendations for selection of seasonal influenza vaccine for children $>6$ months to $<5$ years, adults and other risk groups.

http://www.healthemergency.gov.au/internet/healthemerg ency/publishing.nsf/Content/2723D54E78BF5739CA257 6400027E1E9/\$File/atagi-TIV-30jul10.pdf

[33] Centers for Disease Control and Prevention (2010) Media statement: ACIP recommendation for use of CSL influenza vaccine.

http://www.cdc.gov/media/pressrel/2010/s100806.htm

MDV: Multi-dose vials;

RCH: Royal children's hospital, Melbourne, Victoria; SAEFVIC: Surveillance of adverse events following immunisation in the community;

TGA: Therapeutic goods administration;

TIV: Trivalent influenza vaccines. 